\title{
ANÁLISE DA SITUAÇÃO EPIDEMIOLÓGICA DA HANSENÍASE EM UM MUNICÍPIO DO NORTE DO BRASIL NO PERÍODO 2017-2018
}

\author{
ANALYSIS OF THE EPIDEMIOLOGICAL SITUATION OF LEPROSY IN A \\ MUNICIPALITY IN THE NORTH OF BRAZIL - PERIOD 2017-2018
}

\author{
Lara Inês Martins Dantas ${ }^{1}$, Felipe Vanderley Nogueira ${ }^{1}$, Marlla Christina \\ Ribeiro Gomes ${ }^{1}$, Andriele Gasparetto ${ }^{2}$.
}

\begin{abstract}
RESUMO
Introdução: O Brasil é o segundo país com maior número de hansenianos do mundo, tendo o estado do Tocantins como a segunda posição brasileira. Dos municípios do Tocantins, Porto Nacional está entre os quatro que registraram maior número de casos novos da doença. Com base no exposto, esse trabalho teve como objetivo verificar a incidência de Hanseníase e perfil dos doentes no município de Porto Nacional/TO no período de 2017 e 2018. Metodologia: Trata-se de um estudo epidemiológico, de natureza descritiva do tipo transversal, desenvolvido no município de Porto Nacional/TO, com informações obtidas no Sistema de Informação de Agravos de Notificação (SINAN), da Secretaria de Saúde de Porto Nacional no período de 2017-2018, coletadas no mês de maio de 2019 , pelas autoras da presente pesquisa, com posterior análise estatística no software Excel. Resultados: No ano de 2017 houve 52 notificações, enquanto que em 2018 esse número aumentou para 62. A unidade de saúde com maior número de diagnósticos, aproximadamente $70 \%$, foi o serviço ambulatorial especializado, assim como o modo de detecção mais comum foi o encaminhamento. Discussão: comparando os resultados com outros estudos obteve-se que a hanseníase não tem um padrão de ocorrência por sexo, Já a prevalência da classificação multibacilar em estudos de diversas regiões, está associada ao diagnóstico tardio, o que é reflexo de que os serviços de saúde do Brasil ainda não estão conseguindo captar e tratar todos os casos precocemente. Conclusões: Os dados mostraram que é preciso garantir a melhoria dos serviços de saúde, especialmente no que diz respeito à atenção básica. Além de incentivar os profissionais de saúde a realizar as devidas notificações e dessa forma garantir o subsídio para a tomada de decisão.
\end{abstract}

Palavras-chave: Hanseníase; Medidas em Epidemiologia; Atenção Primária à Saúde. ciências da saúde pela Universidade Federal do Rio Grande - FURG, professora da disciplina Interação Ensino Serviço e Comunidade do Instituto Tocantinense Presidente Antônio Carlos - ITPAC.

Autor correspondente: Lara Inês Martins Dantas.Porto Nacional, Tocantins, Brasil; Endereço: Rua Phetion Pereira Lima Jardim dos Ipês, Qd- 12, Lt- 04 C2; telefone: (94) 99198-9887; e-mail: larainesdantas@hotmail.com

Editor: Carvalho A. A. B. Medicina, Universidade Federal do Tocantins, Brasil.

Publicado: 23 de fevereiro de 2021.

Direitos Autorais: (c) 2021 Dantas et al. Este é um artigo de acesso aberto que permite o uso, a distribuição e a reprodução sem restrições em qualquer meio, desde que o autor original e a fonte sejam creditados.

\begin{abstract}
Introduction: Brazil is the second country with the highest number of leprosy patients in the world, with the state of Tocantins as the second Brazilian position. Of the municipalities of Tocantins, Porto Nacional is among the four that registered the highest number of new cases of the disease. Based on the above, this study aimed to verify the incidence of Hansen's disease and patient profile in the city of Porto Nacional / TO in the period 2017 and 2018. Methodology: This is a cross-sectional, epidemiological study, developed in the municipality of Porto Nacional / TO, with information obtained in the Information System for Notifiable Diseases (SINAN), of the Health Department of Porto Nacional in the period 2017-2018, collected in May 2019, by the authors of this research, with subsequent statistical analysis in Excel software. Results: In 2017, there were 52 notifications, while in 2018 this number increased to 62 . The health unit with the highest number of diagnoses, approximately $70 \%$, was the specialized outpatient service, as well as the most common mode of detection was forwarding. Discussion: comparing the results with other studies, it was found that leprosy does not have a pattern of occurrence by sex, whereas the prevalence of multibacillary classification in studies in different regions is associated with late diagnosis, which is a reflection of the fact that health in Brazil are still unable to capture and treat all cases early. Conclusions: The data showed that it is necessary to guarantee the improvement of health services, especially with regard to primary care. In addition to encouraging health professionals to make the necessary notifications and thus guarantee the subsidy for decision making. Keywords: Leprosy; Epidemiologic Measurements; Primary Health Care.
\end{abstract}


INTRODUÇÃO

A Hanseníase é uma doença infectocontagiosa de notificação compulsória e investigação obrigatória em todo território nacional e possui como agente causador o Mycobacterium leprae (M. leprae). É uma patologia de evolução crônica que acomete a pele e o sistema nervoso periférico, manifestando-se com diminuição de sensibilidade, sendo curável, de baixa contagiosidade e contra a qual a maioria da população tem defesa imunológica natural, mas se não diagnosticadas e tratadas adequadamente, podem evoluir para incapacidades físicas permanentes ${ }^{1}$.

Historicamente, a Hanseníase foi conhecida como Lepra, uma das doenças mais antigas que acometeram o homem. Há evidências objetivas desta datando do segundo século antes de Cristo, mas foi por volta do ano 150 depois de Cristo que essa patologia tornou-se bem conhecida, uma vez que era transferida entre os países por soldados infectados, comerciantes e colonizadores. No Brasil, os primeiros casos da Hanseníase foram notificados no ano de 1600, mas apenas em 1912 destacou-se a importância das notificações compulsórias e de se tratar essa doença com rigor científico. Por volta de 1916, foi instituída, na cidade do Rio de Janeiro, a Comissão de Profilaxia da Lepra, sendo esse um marco para que o governo adotasse cada vez mais medidas para a patologia ${ }^{2,3}$.

Atualmente, o Brasil é o segundo país com maior número de hansenianos do mundo, ficando atrás somente da Índia, a qual tem uma população cinco vezes maior que o Brasil. Dos estados brasileiros, o Tocantins tem ocupado a segunda posição, considerando a Hanseníase como hiperendêmica ${ }^{4,5}$. E dos municípios do Tocantins, Porto Nacional está entre as quatro cidades que registraram maior número de casos novos da doença ${ }^{6}$.

Tendo em vista a importância epidemiológica exibida pelo município de Porto Nacional em relação ao controle da endemia no Estado do Tocantins, esse trabalho tem como objetivo verificar a incidência de Hanseníase e perfil dos doentes no município de Porto Nacional/TO no período de 2017 e 2018.

\section{METODOLOGIA}

Trata-se de um estudo epidemiológico, de natureza descritiva e do tipo transversal. Este trabalho foi desenvolvido em Porto Nacional/TO, dada à relevância epidemiológica apresentada pelo município em relação às notificações no Estado do Tocantins. Para realização da pesquisa usou-se as informações obtidas no Sistema de Informação de Agravos de Notificação (SINAN) disponibilizadas pela Secretaria de Saúde de Porto Nacional, coletadas no mês de maio de 2019 pelas autoras da presente pesquisa. Os critérios de inclusão foram: hansenianos diagnosticados, residentes no município de Porto Nacional e notificados no SINAN. As variáveis analisadas foram: o sexo, a faixa etária, a raça, o bairro residencial, a unidade de saúde que realizou a notificação, a classificação operacional da doença, a forma clínica, o esquema terapêutico, o modo de deteç̧ão e o modo de entrada. Para análise estatística, os dados foram lançados no software Excel, no qual foi feita a comparação dos resultados epidemiológicos de 2017 e 2018.

\section{RESULTADOS}

No ano de 2017 houveram 52 notificações, enquanto que em 2018 esse número aumentou 19\% indo para 62 notificações. A partir desses relatórios, observou-se que o sexo mais acometido, tanto no ano de 2017 como 2018, foi o masculino, sendo que no ano de 2017 a diferença entre os sexos foi de 5,2\% e em 2018 de 0,03\% (TABELA 1). Em relação à zona residencial mais frequente, tanto no ano de 2017 como 2018 foi a urbana, mas se analisar os bairros separadamente, a zona rural se sobrepõe (TABELA 1). Dentre as unidades de saúde com maior número de casos diagnosticados, encontrouse com $70 \%$ o serviço ambulatorial especializado.

Tabela I - Distribuição dos registros, segundo sexo e zona de residência, entre os casos de hanseníase, Porto Nacional-TO, 2017-2018.

\begin{tabular}{|l|l|l|}
\hline Caracteristica & $\mathbf{2 0 1 7}$ & $\mathbf{2 0 1 8}$ \\
\hline Sexo & 31 & 32 \\
\hline Masculino & 21 & 30 \\
\hline Feminino & 52 & 62 \\
\hline Total & \multicolumn{2}{|l|}{} \\
\hline Zona de residência & 46 & 53 \\
\hline Urbana & 6 & 9 \\
\hline Rural & 52 & 62 \\
\hline Total
\end{tabular}

A raça mais notificada nos dois anos foi a parda, em um total de 71\% em 2017 e 77,5\% em 2018. Em relação à classificação operacional da doença a maior incidência, nos dois anos consecutivos, foi de multibacilar com $77 \%$ dos casos, sendo a dimorfa a forma clínica mais comum (TABELA 2).

Tabela II - Distribuição dos registros, segundo, raça/cor, classificação operacional atual e forma clínica entre os casos de hanseniase, Porto Nacional-TO, 2017-2018. Raça/cor

\begin{tabular}{|l|l|l|}
\hline Ignorada/branca & 2 & 6 \\
\hline Branca & 4 & 1 \\
\hline Preta & 8 & 5 \\
\hline Amarela & 1 & 2 \\
\hline Parda & 37 & 48 \\
\hline Total & 52 & 62 \\
\hline
\end{tabular}

\section{Classificação operacional atual}

\begin{tabular}{|l|l|l|}
\hline Paucibacilar & 14 & 12 \\
\hline Multibacilar & 38 & 50 \\
\hline Total & 52 & 62 \\
\hline Forma clinica
\end{tabular}

Forma clínica

\begin{tabular}{|l|l|l|}
\hline Indeterminada & 11 & 12 \\
\hline Tuberculóide & 4 & 8 \\
\hline Dimorfa & 32 & 36 \\
\hline Virchowiana & 2 & 1 \\
\hline Não Classificada & 3 & 5 \\
\hline Total & 52 & 62 \\
\hline
\end{tabular}

Em relação ao modo de detecção, o mais comum, também nos dois anos, com $50 \%$ das notificações, foi o encaminhamento (TABELA 3). Não foi avaliado o modo de saída, pois este depende do tempo de tratamento do paciente. 
Tabela III - Distribuição dos registros, segundo o modo de detecção entre os casos de hanseníase, Porto Nacional-TO, 2017-2018.

Modo de detecção

\begin{tabular}{|l|l|l|}
\hline Ignorada/branca & 9 & 13 \\
\hline Encaminhamento & 31 & 27 \\
\hline Demanda espontânea & 5 & 8 \\
\hline Exame coletividade & 0 & 2 \\
\hline Exame contato & 7 & 10 \\
\hline Outros modos & 0 & 2 \\
\hline Total & 52 & 62 \\
\hline
\end{tabular}

\section{DISCUSSÃO}

Comparando os resultados deste estudo com o trabalho de Neves ${ }^{11}$,o qual avaliou a situação da Hanseníase em Palmas, no período entre 2005 e 2010, houveram 1.362 indivíduos notificados em que se observaram resultados semelhantes aos obtidos em Porto Nacional, ou seja, sua maioria foi paciente do sexo masculino, e que eram multibacilares da forma clínica dimorfa.

Esses resultados são apoiados pela pesquisa de Marques et al ${ }^{12}$ em Tangará da Serra, estado de Mato Grosso, que também teve maior incidência de casos no sexo masculino, com predomínio de classificação multibacilar da forma clínica dimorfa. Já em relação à raça, a mais afetada nesse município é a branca com $43 \%$, porém a parda apresenta $41 \%$ das notificações.

No estudo de Brito et al $^{13}$ na cidade de Fortaleza no Ceará, a proporção de casos novos foi crescente no sexo masculino, com prevalência da classificação multibacilar com aumento progressivo nos últimos anos dos casos classificados na forma clínica virchowiana.

Apesar de esses estudos mostrarem uma porcentagem um pouco maior no sexo masculino, há outros trabalhos como o de PINTOA et $a^{14}$ que referem maior detecção no sexo feminino, o que comprova que a Hanseníase não tem um padrão de ocorrência por sexo ${ }^{13}$.

Já a prevalência da classificação multibacilar em estudos de diversas regiões, está associada ao diagnóstico $\operatorname{tardio}^{13}$, o que é reflexo de que os serviços de saúde do Brasil ainda não estão conseguindo captar e tratar todos os casos precocemente.

Em Porto Nacional, isso pode ter relação com os dados epidemiológicos, que despontam que a maioria dos casos (70\%) tem como método de detecção o encaminhamento e o maior número de casos é notificado pelo serviço ambulatorial especializado. Mostra-se, assim, que é preciso garantir a melhoria dos serviços de saúde, especialmente no que diz respeito à atenção básica, garantindo a ampliação da descentralização das ações como estratégia fundamental para se reduzir a carga da doença, principalmente nas áreas com maior concentração de casos.
Em Porto Nacional, há uma grande concentração de casos na zona rural, quando se compara a zona urbana dividida em bairros. O que, pode ser relacionado há um local com dificuldade de acesso ao sistema de saúde e diagnóstico em fases avançadas. Isso é corroborado pelo estudo de IMBIRIBA et al (15) em Manaus no Amazonas, que diz que apesar de a espacialização da doença ser imprecisa nessa região, ela tem relação com a condição de vida precária e consequentemente falta de assistência a saúde.

\section{CONCLUSÃO}

Com base no exposto, pode-se indeferir que o perfil de acometimento da hanseníase em Porto Nacional é, sobretudo em pacientes do sexo masculino, de raça parda, residente na zona urbana, com classificação operacional multibacilar, forma clinica dimorfa e detectado por encaminhamento.

Tendo isso em vista, é relevante priorizar ações de educação em saúde, vigilância de contatos e qualificação do diagnóstico, incentivando os profissionais de saúde a realizar as devidas notificações e dessa forma garantir o subsidio para a tomada de decisão.

\section{REFERÊNCIAS BIBLIOGRÁFICAS}

1. Lastória, J. C., \& Abreu, M. A. M. M. (2012). Hanseníase: diagnóstico e tratamento. Diagn Tratamento, 17(4), 173-9. Edit, L. M. (2004). Breve história da hanseníase: sua expansão do mundo para as Américas, o Brasil eo Rio Grande do Sul e sua trajetória na saúde pública brasileira. Saude Soc, 13, 76-88.

2. de Souza-Araujo, H. C. (1933). Contribuição à epidemiologia e prophylaxia da lepra no Norte do Brasil. Memórias do Instituto Oswaldo Cruz, 27(3), 165-337.

3. Sales, O. P., dos Santos Martins, F. J., \& Lopes Da Cruz Amaral, J. B. (2018). LEPROSY PROBLEM OF PUBLIC HEALTH IN TOCANTINS: WHAT REVEALS THE PUBLIC DOMAIN DATA OF 2011-2015. HUMANIDADES \& INOVACAO, 5(2), 272-281.

4. Monteiro, L. D., Martins-Melo, F. R., Brito, A. L., Lima, M. D. S., Alencar, C. H., \& Heukelbach, J. (2015). Tendências da hanseníase no Tocantins, um estado hiperendêmico do Norte do Brasil, 2001-2012. Cadernos de Saúde Pública, 31, 971-980.

5. BRASIL - Ministério da Saúde. Projeto inovador para o enfrentamento da hanseníase é realizado em Tocantins. 2017 [acesso em: 2019 jun 10]. Disponível em: http://www.saude.gov.br/noticias/agencia-saude/41645projeto-inovador-para-o-enfrentamento-da-hanseniase-erealizado-em-tocantins

6. Freitas, L. R. S. D., Duarte, E. C., \& Garcia, L. P. (2017). Análise da situação epidemiológica da hanseníase em uma área endêmica no Brasil: distribuição espacial dos períodos 2001-2003 e 2010-2012. Revista Brasileira de Epidemiologia, 20, 702-713.

7. MS/SVS-Ministério da Saúde/Secretaria de Vigilância em Saúde. (2016). Diretrizes para vigilância, atenção e eliminação da Hanseníase como problema de saúde pública: manual técnico-operacional.

8. Magalhães, M. D. C. C., \& Rojas, L. I. (2007). Diferenciação territorial da hanseníase no Brasil. Epidemiologia e Serviços de Saúde, 16(2), 75-84. 
9. Sousa, R. L., Brito, R. R. L., \& Silva, Z. S. S. B. (2012). Dificuldades encontradas pelos enfermeiros (as) das ubs de uma cidade do Tocantins frente à prevenção de incapacidades em hanseníase. Revista Científica do ITPAC, Araguaína, 5(4).

10. Neves, T. V., Valentim, I. M., Vasconcelos, K. B., Rocha, E. S. D., Nobre, M. D. S. R. S., \& Castro, J. G. D. (2013). Perfil de pacientes com incapacidades físicas por hanseníase tratados na cidade de Palmas-Tocantins. Gestão e Saúde, 4(2), 2016-2025.

11. Marques, M. S., Cabral, J. F., Terças, A. C. P., Santana, D. P., \& da Silva, J. H. (2018). Perfil clínico e epidemiológico da hanseníase no município de Tangará da Serra, Mato Grosso. Renome, 6(2), 34-47.

12. Brito, A. L., Monteiro, L. D., Ramos Junior, A. N., Heukelbach, J., \& Alencar, C. H. (2016). Tendência temporal da hanseníase em uma capital do Nordeste do Brasil: epidemiologia e análise por pontos de inflexão, 2001 a 2012. Revista Brasileira de Epidemiologia, 19, 194-204.

13. Pinto RA, Maia HF, Silva MAF, Marback M. Perfil Clínico e Epidemiológico dos Pacientes Notificados com Hanseníase em um Hospital Especializado em Salvador, Bahia. Rev. B. S. Publica Miolo. out/dez 2010;34(4):906-918.

14. Imbiriba, E. N. B., Silva Neto, A. L. D., Souza, W. V. D., Pedrosa, V., Cunha, M. D. G., \& Garnelo, L. (2009). Socia inequality, urban growth and leprosy in Manaus: a spatial approach. Revista de saude publica, 43(4), 656-665. 